DOI:10.22374/jeleu.v3i3.93

\title{
PERFORMANCE OF URODYNAMICS DURING THE COVID-19 PANDEMIC: A QUESTIONNAIRE SURVEY
}

\author{
Talal Jabbar ${ }^{1}$, Samuel Mills ${ }^{2}$, Richard Simpson ${ }^{3}$, Adam Jones ${ }^{3}$, lain Campbell ${ }^{4}$, Omar Al-Mula Abed ${ }^{4}$ \\ ${ }^{1}$ Consultant Urologist, North Bristol NHS Trust \\ ${ }^{2}$ Educational Fellow in Urology, East Lancashire Hospitals NHS Trust \\ ${ }^{3}$ Specialty Registrars, East Lancashire Hospitals NHS Trust \\ ${ }^{4}$ Consultant Urologist, East Lancashire Hospitals NHS Trust
}

Correspondence: omar.mulaabed@gmail.com

Submitted: July 8, 2020. Accepted: July 15, 2020. Published: August 21, 2020.

\begin{abstract}
Aims

The principal aim of this multicentre, international cohort study is to explore the safety concerns of clinicians when performing urodynamic studies (UDS) during the COVID-19 pandemic. This study will also assess provider preference on personal protective equipment (PPE) during UDS and awareness of relevant international guidelines. The outcome is to offer a practical means of reducing the risk of aerosol transmission during UDS, to include a protocol for screening patients, and to consider safer methods of inducing urethral leak-point pressure.
\end{abstract}

\section{Methods}

A 14-point, multiple-response, online questionnaire was designed through Survey Monkey. This was distributed to healthcare workers involved in UDS via email, WhatsApp, and Twitter. This included, of varying experience; urologists, urogynaecologists, clinical nurse specialists, and clinical scientists across the United Kingdom (UK), United States of America (USA), Australia, Europe, and Middle-East.

\section{Results}

The study was conducted over two weeks and there were 104 responses to the survey. The majority of respondents were consultants (60\%) and $73 \%$ of respondents performed UDS regularly. There were $56.7 \%$ of respondents who felt that UDS was safe to be re-instated and there were $52.8 \%$ who did not feel coughing was safe during the procedure. At the time of the survey, $83.5 \%$ were unaware of any national or international urological guidelines on the best practice for urodynamics during this era and $52.8 \%$ of clinicians stated they would wear PPE during UDS if it were reinstated.

\section{Conclusion}

The majority of respondents felt safe to reinstate UDS; however, they felt more official guidance and recommendation is needed. Our proposed protocol can provide a safe and practical approach that keeps healthcare workers and patients safe.

Keywords: Urodynamics, COVID-19 
The lower urinary tract is modulated by a complex neuromuscular system and anatomy which can be difficult to understand. The introduction of urodynamic studies (UDS) has become a useful procedure to aid the diagnosis of lower urinary tract pathology. This includes stress urinary incontinence, detrusor overactivity, bladder outlet obstruction, and detrusor underactivity. Also, UDS has a role in monitoring the progress of a disease, mainly in the neuropathic bladder; and objectively evaluating the response to various therapies. It can give an accurate understanding of the mechanical behaviour of the lower urinary tract, although this is not entirely straightforward, especially if not performed appropriately to a high standard.

When performing UDS, the intravesical pressure ( $p$ ves) is recorded by insertion of a catheter into the bladder. Similarly, the pressure within the rectum is measured and is taken to be representative of abdominal pressure ( $p$ abd). These pressures, alongside uroflow rate, and the volume of fluid infused are recorded during the filling and voiding phases. Patients will be asked to do several cough tests for quality control and to assess for urine leaks. Other maneuvers can be performed including the Valsalva test or more patient-specific movements. ${ }^{1}$

The awareness and control of communicable disease is a pertinent issue. On 7th January 2020, Chinese authorities identified a novel coronavirus with high transmissibility. ${ }^{2}$ The coronavirus, later known as SARS-CoV-19, caused a respiratory disease that spread rapidly across international borders. The coronavirus disease, termed COVID-19, was associated with high morbidity and was declared as a global health emergency by the World Health Organisation (WHO) in late-January $2020 .^{3}$ The spread of disease is believed to occur from the aerosol transmission of respiratory droplets. ${ }^{4}$ To date, there are ten million diagnosed cases of COVID-19 worldwide, with over half a million recorded deaths. ${ }^{5}$ This has caused a significant impact on health care systems and has prompted the European Association of Urology (EAU) to recommend deferring some non-urgent urological procedures. ${ }^{6}$ However, as we are now entering the recovery phase of this pandemic, consideration should be made to reinstate certain procedures safely, such as UDS.
UDS is performed with patients and clinicians in close proximity. It is an interactive procedure which requires several adjustments and intervention. A cough test is frequently repeated as part of the study and this aerosol-generating action may increase the risk of viral transmission (7). This process is repeated to ensure device calibration and for assessment of urethral sphincter competence. In this context, the test is referred to as the cough-induced leak point pressure (CLPP). Albeit a valuable diagnostic test, the routine assessment of CLPP requires careful consideration in environments with a high prevalence of the communicable disease. Coughing permits the rapid dissemination of aerosol particles and could act as a conduit for the aerosol transmission of disease. ${ }^{7}$

An alternative means of assessing for urethral sphincter competence is the use of the Valsalva maneuver. Although both methods are effective at increasing intra-abdominal pressure, the urethral leak-point pressure is more frequently induced with coughing. ${ }^{8}$ Also, most practicing urologists did not use the Valsalva as a standard for calibration in the pre-pandemic days.

The EAU urinary incontinence guidelines panel recently recommended the deferral of UDS during the COVID-19 outbreak. $^{6}$ At the time of writing, there is no published strategy on when, or how, this valuable diagnostic test can be restarted. This study seeks to investigate the safety concerns of clinicians in providing UDS in a COVID-19 era. It aims to explore clinician awareness of existing guidelines and opinions on the use of personal protective equipment (PPE). Authors will offer practical means of reducing the risk of disease transmission and propose a patient screening protocol in preparation for a future scenario where guidelines recommend restarting UDS.

\section{METHODOLOGY}

A 14-point online questionnaire was designed using Survey Monkey. It was distributed directly to urologists, urogynaecologists, clinical nurse specialists, and clinical scientists across the UK, USA, Australia, Europe, and Middle-East via email, WhatsApp and Twitter. The survey was open for two weeks from 27/05/2020. Respondents were asked to give details of their level of experience in performing UDS and 
the type of institution they work in. Questions aimed to assess how prepared responders felt for re-instating UDS and any concerns related to this. The survey questioned the willingness of respondents to allow patients to cough and whether the Valsalva maneuver was a safer alternative without compromising the accuracy of UDS.

\section{RESULTS}

There were 104 responses to the survey during the two weeks. All responders were members of either the British Association of Urological Surgeons (BAUS), the Society of Urodynamics, Female Pelvic Medicine \& Urogenital Reconstruction (SUFU), the International Continence Society (ICS) or the Urological Society of Australia and New Zealand (USANZ). The majority of respondents $(60 \%)$ were Consultants in urology or urogynaecology (Figure 1 'Q4'). Of the 104 respondents, 73\% perform UDS routinely, and $74 \%$ have it identified as part of their job plan. The responses were from different types of institutions (DGH 18.2\%; Teaching Hospital 43.2\%; Tertiary Centre $38.4 \%$ ).

The majority of respondents felt safe in reinstating UDS at the time of asking (56.7\%), with only $16.3 \%$ stating they would not; the remaining $26.9 \%$ were unsure (Figure 2 'Q5'). Contrary to these findings only $16.3 \%$ would feel safe allowing a patient to cough during the procedure, $52.8 \%$ would not; $30.7 \%$ were unsure (Figure 3 'Q6').

FIG. 1 Respondent (104) job titles.

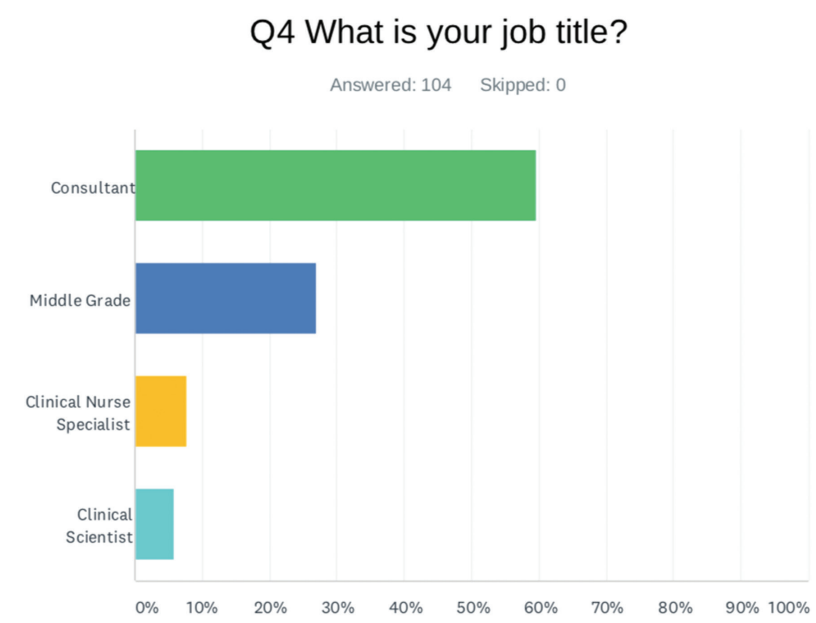

FIG. 2 Respondent (104) opinions regarding whether they felt safe to reinstate urodynamic studies within the COVID-19 pandemic.

\section{Q5 Do you feel safe re-instating this test?}

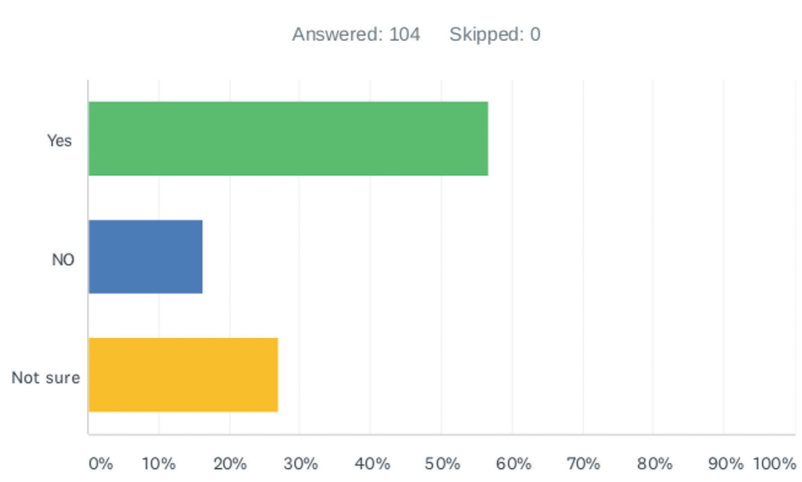

FIG. 3 Responses (104) to whether clinicians would feel safe allowing the patient to cough during the procedure.

Q6 In the current era of Covid-19, do you feel it is safe to ask the patient to cough during the test? Answered: 104 Skipped: 0

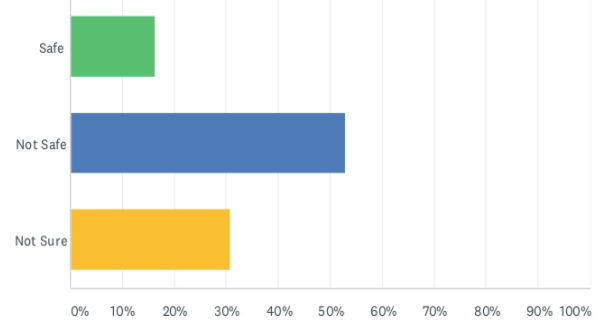

To assess alternative methods of performing UDS during the pandemic, we enquired whether respondents consider the Valsalva maneuver a suitable substitute for coughing. The majority (44.2\%) consider this satisfactory, $24 \%$ wound not, and $31.7 \%$ were unsure (Figure 4 'Q7'). If UDS was to be reinstated 52.8\% of respondents would wear 'full PPE' during the procedure (NP95 face mask, eye protection, surgical gown) (Figure 5 'Q8'). The majority of respondents were not aware of formal guidance specifically concerning UDS from regulatory bodies (No 55.7\%; Unsure 27.8\%; Yes 16.3\%).

Before the COVID-19 pandemic, $48 \%$ of respondents would perform UDS on over 6 patients per week 
FIG. 4 Respondents (104) likeliness to wear full PPE for all patients undergoing urodynamic studies should it be reinstated.

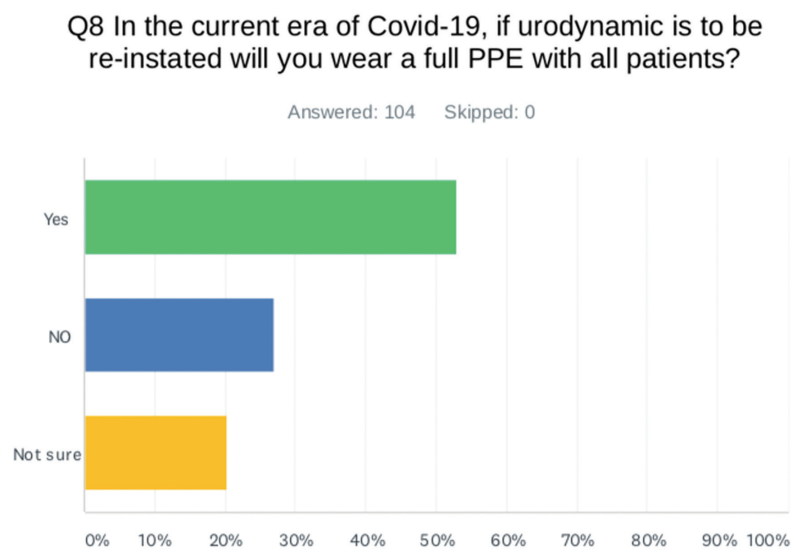

FIG. 5 Respondents (104) opinions regarding the suitability of the Valsalva maneuver as an alternative to coughing.

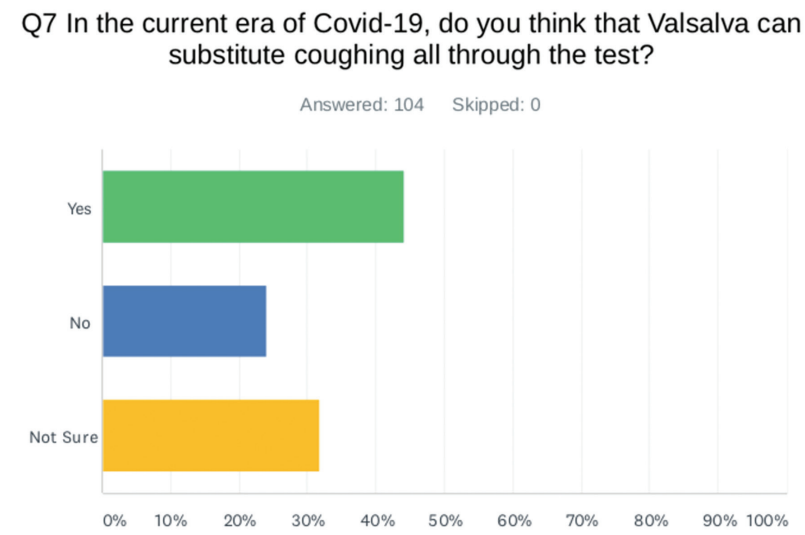

(Figure 6 'Q9'). Following the COVID-19 pandemic, $63.4 \%$ do not plan to reinstate the same number of appointments to each clinic (Figure 7 'Q10'). Air circulation systems are not found in $51.4 \%$ of rooms in which UDS is performed by the respondents.
Respondents were given the chance to state any other concerns, or suggest any stipulations, before restarting UDS of which 77/104 (74.0\%) and 71/104 $(68.2 \%)$ respondents replied, respectively. There were $51 / 104(49.0 \%)$ that had no concerns. Two responders stated that they did not have concerns and specified low infection rates of COVID-19 being the reason.

FIG. 6 The average number of patients on which urodynamic studies is performed by 104 respondents per week.

Q9 On average, how many patients do you see per week? Answered: 104 Skipped: 0

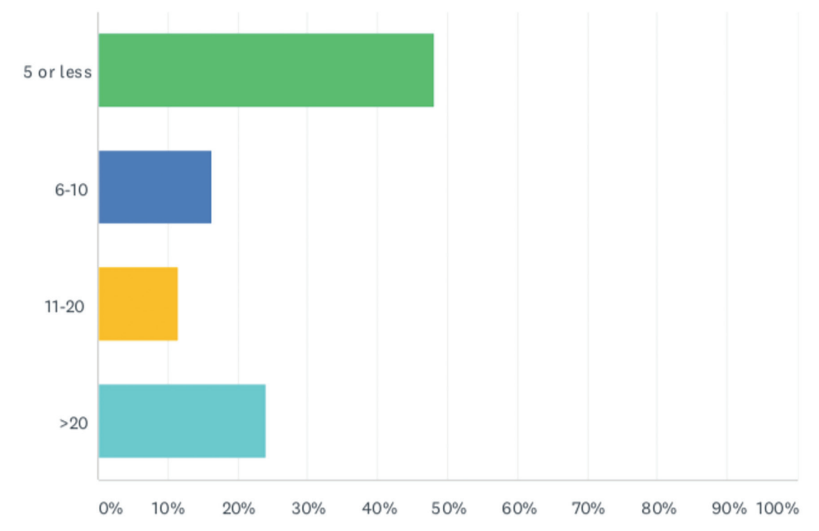

FIG. 7 Respondents (104) were asked whether they would reinstate as many urodynamic studies appointments compared to the pre-COVID-19 era.

Q10 Would you reinstate the same number of patients as pre- Covid-19? Answered: 104 Skipped: 0

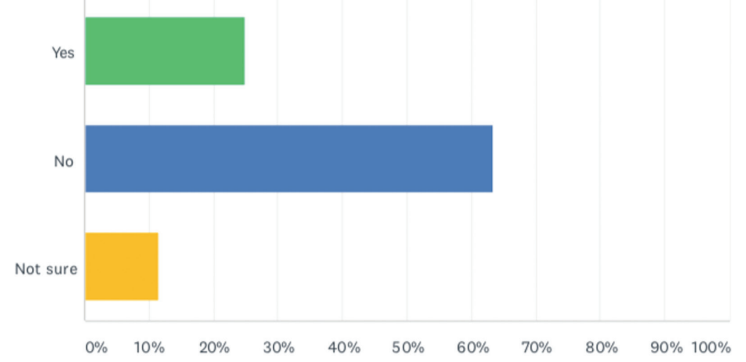

J Endolum Endourol Vol 3(3):e13-e21; August 21, 2020.

This article is distributed under the terms of the Creative Commons Attribution-Non Commercial 4.0 International License. (c) Jabbar, et al. 
The survey allowed the participant to express their feelings within a free text column and the most common concerns are listed below.

- The patient and the doctor should both wear a mask so that the patient may cough safely.

- There may be the option of having to self-isolate and have a negative COVID-19 test before doing urodynamics.

- Risk of the rectal probe and the known presence of the virus in stool.

- The patient might develop COVID-19 post-test.

- Patient's non-compliance with infection control policy, especially not wearing masks during cough test.

- High-risk patients especially neuropathic patients who are wheelchair-bound with significant comorbidity, size of the urodynamics room, and number of patients in the waiting room.

- Some suggested bearing down is preferable to Valsalva.

They were asked about their recommendations and suggestions before restarting UDS and most common comments are listed below.

- Pre-urodynamic COVID-19 testing of patients with a risk scoring system for patients and clinicians.

- Robust telephone triage before appointment to ensure patients have no COVID-19 symptoms or contact with confirmed cases.

- Defer UDS in vulnerable patients unless is clinically urgent.

- Adequately spaced appointments for cleaning between patients.

- Clear local protocol, guidelines, and post urodynamics instructions.

- Minimal staff to be present within UDS room to enable safe social distancing.

- Patient should be attended alone if possible.

- The use of screens between patient and staff or remote control to UDS machine.

\section{DISCUSSION}

Several authorities and healthcare systems have taken unprecedented measures to contain the spread and surge in the prevalence of patients with COVID-19. Initially, strict healthcare measures were adopted, such as the suspension of non-urgent elective surgery and the limitation of inpatient and outpatient services. The use of PPE has increased and becomes more vigilant whilst performing any diagnostic procedures as well as in the surgical theatre. Some healthcare systems have started checking temperatures and symptom screening for patients coming to the hospitals; others are performing COVID-19 tests before invasive procedures. ${ }^{9,10}$ Subsequently, these measures were escalated to include a recommended 14 days quarantine with self-isolation at home for patients pre- and postinvasive procedures. ${ }^{17}$ Following general government guidance, the use of face masks is protective for both healthcare workers and people in the community exposed to infection and wearing face masks is now acceptable and feasible. As the national pandemic is in the recovery phase, many of the urological diagnostic and therapeutic procedures, including UDS, may be reinstated shortly.

Worldwide, UDS procedures are standardized according to an agreed protocol defined in the ICS guidelines on good urodynamic practice. ${ }^{11}$ The standard practice recommendation for patients undergoing this test stipulates that coughs are required for a signal check and transducer calibration at the start of the procedure. Following this, regular 'test coughs' are vital for quality control every $1-2$ minutes or every $100 \mathrm{~mL}$ of infusion to ensure that the lines/transducers are still accurately measuring the change in pressure as the bladder is being filled. ${ }^{12}$ Furthermore, a patient will be asked to cough to look for stress incontinence and assess the urodynamic abdominal leak point pressure (ALPP). Bladder filling is stopped when the patient can no longer tolerate the infusion.

Based on the descriptions above, for an average 5 minutes test, the patient will cough a minimum of 10 times (1-2 for pre-infusion calibration, 5 times for quality checks over 5 minutes, $2-3$ times to check for SUI and ALPP and finally the last coughs before and after the voiding phase). As such there were concerns raised by the urological community as to whether this procedure is safe to perform and if Valsalva could be a suitable substitute to the cough test. Our survey found that, at the time of asking, only $16 \%$ of clinicians 
would be happy to allow a patient to cough during the procedure (see Figure 3 ' $\mathrm{Q} 6$ ').

Furthermore, we should question whether it is right to perform a diagnostic test which will generate an increased number of lower urgency elective procedures at a time when more priority should be given to catching up with urgent cancer services.

Clinicians in our survey were mostly satisfied that Valsalva could replace coughing; however, a third of respondents stated they were unsure (see Figure 4 'Q7') and as recommended, the majority of clinicians in our survey (52\%) would want to wear full PPE if they were to reinstate UDS in their institution. Hashim et al suggests general measures such as the use of PPE for all personnel in the room and maintaining a 2 metre distance ${ }^{10}$ albeit the latter is controversial and could change in the future. Our analysis couldn't clarify as to whether the PPE will include a surgical mask versus and FFP3 mask in addition to the standard apron, gown, gloves, and visors but there was a more specific suggestion and alterations aim to reduce the frequency of coughing and require a change to how UDS is normally performed. This could be possible by substituting coughing for Valsalva or gentle external pressure on the bladder. If the patient should need to cough, then this should be directed away from staff and shielded by the patient's elbow or a tissue. ${ }^{10}$

Several studies have looked into cough amplitudes in urodynamic studies and the presence of high-quality cough signals has been recommended as an important component of high-quality urodynamic results. Lu et al have concluded in his 539 urodynamic trace analysis that high-quality traces were observed with type I initial good quality coughs $(\mathrm{P}<0.01)$ and if this cough signal is obtained at the time of calibration, it will be likely observed later on during the test resulting in greater and more accurate results as a reasonable standard. ${ }^{13}$

In 2003, Sullivan et al were the first to explore and propose a grading system for the quality of coughs. ${ }^{12}$ Sulivan et al. divided coughs into grade A, B or C depending on the signal amplitude change they create on the urodynamics trace (>100, 80-99 and 50-79 $\mathrm{cmH} 20$ for each grade respectively). The study audited 100 traces at the time and concluded that despite being a tertiary centre with high volume turnover for this test, significant defects in quality control were not uncommon. Accordingly, coughs are often of variable quality and so Valsalva could theoretically be a safer and possibly equally accurate modality to increase the abdominal pressure during UDS but more reflection, training and higher standard testing calibration should take place for this as an improvement.

The United States government's centre for disease control (CDC) has officially made the emerging scientific evidence on Coronavirus transmission. The virus needs a dose of about 1000 viral particles (vp), to successfully infect a person. The formula for successful infection is (exposure to virus $\times$ time). ${ }^{14}$

The typical environmental spread of activities:

- Breath: $20 \mathrm{vp} /$ minute

- Speaking: $200 \mathrm{vp} /$ minute

- Sneeze: 200 million vp

- Cough: 200 million vp (enough of these may remain in air for hours in a poorly ventilated environment)

At the time the questionnaires were sent out and completed the United Kingdom's government COVID alert level was at 4 indicating that the COVID-19 epidemic is in general circulation and transmission is high or rising exponentially. The recommended action for level 4 was that social distancing measures and restrictions should be continued as it was at level 5 however as we were just writing the final review of this manuscript the government reduced the alert level to 3 indicating that A COVID-19 epidemic is still in general circulation and recommends gradual relaxing of restrictions and social distancing measures. ${ }^{15}$ The information, assessment, and protocols can be used for any other pandemics. On-going service audit to assess the infection rate with COVID-19 following urodynamics as a change in practice or further recommendations should be considered such as pre-test COVID-19 swab test for all patients as for other elective surgical procedures. This point was highly suggested by our survey participants. Other suggestions are selfisolation pre and post-test and updated information leaflets to fit the purpose during this current era.

Public Health England does not classify urodynamics as an aerosol-generating procedure ${ }^{16}$; however, The results of our study found that less than one in five corresponding clinicians felt that it was safe to allow 
participants to cough during urodynamics testing in the current COVID-19 pandemic (see Figure 3 "Q6"). Albeit an established role within urodynamics testing, the act of coughing permits the aerosol transmission of particles. This can expose health workers situated in the same assessment room to potentially infective particles within five minutes of expulsion. ${ }^{7}$ Given the highly transmissible nature of COVID-19, it is understandable that this may cause safety concerns for providers of urodynamics. Respondents in our study have shown concerns regarding reinstating UDS in the current climate (see Figure 2 ' $\mathrm{Q} 5$ )

In relation to reducing the spread of disease, $52.8 \%$ of responding clinicians cited the need for contact precautions and safe use of PPE while $20.1 \%$ were not sure. Contact precautions may be incorporated into routine UDS through the use of telehealth triage, screening, and use of ambulatory urodynamics testing. A practical solution to triaging patients would be to offer a telephone consultation. This can be used to determine the severity of lower urinary tract symptoms, urgency for testing and, opportunistically, screening for symptoms or contacts relating to COVID-19. A potential screening protocol is outlined in Appendix 1. The authorship has developed a pre-test assessment form that can be utilized to aid the triage patients on waiting lists for UDS. This could be completed by a health care professional over the phone or can be sent out to patients with the standard procedure leaflet, bladder diary chart, and relevant ICIQ questionnaires. This form should be completed and returned to the urodynamic unit at least a week before the proposed test.

The form will collect demographic data from patients in addition to several other factors by which a risk score could be built up. It is important to know if the patient is shielding or living with someone who is shielding in which case the patient should not attend and the procedure should be deferred. If they are symptomatic (continuous cough in the last seven days, difficulty breathing, loss of sense of smell or taste) or living with someone unwell with these symptoms recently. Moving onto other factors that have been identified as important in the decision making as if the patient has experienced an improvement in his/her urological symptoms (if yes, they can be removed from the pathway and reviewed routinely in the outpatient clinic). Other important information needed is those to assess the general well being and any other comorbidities risks which make the patient more vulnerable and at higher risk (Table 1). The risk is categorized into low risk (green), moderate risk (amber), and high risk (red). Those who fall in the moderate risk can be further prioritized according to clinical urgency and need. Hashim et al have clarified these categories as P2 (to be done within 4 weeks), P3 (can be delayed up to 3 months) and P4 (can be delayed beyond 3 months). There is no category P1 (emergency or urgent) in routine elective urodynamics. ${ }^{10}$ The authors also recommend that should the service be reinstated then greater respect to timing slots should be considered and seating spaces in the waiting areas should comply with social distancing guidance set by the government (Figure 8).

FIG. 8 Flow chart of urodynamic studies triaging process.

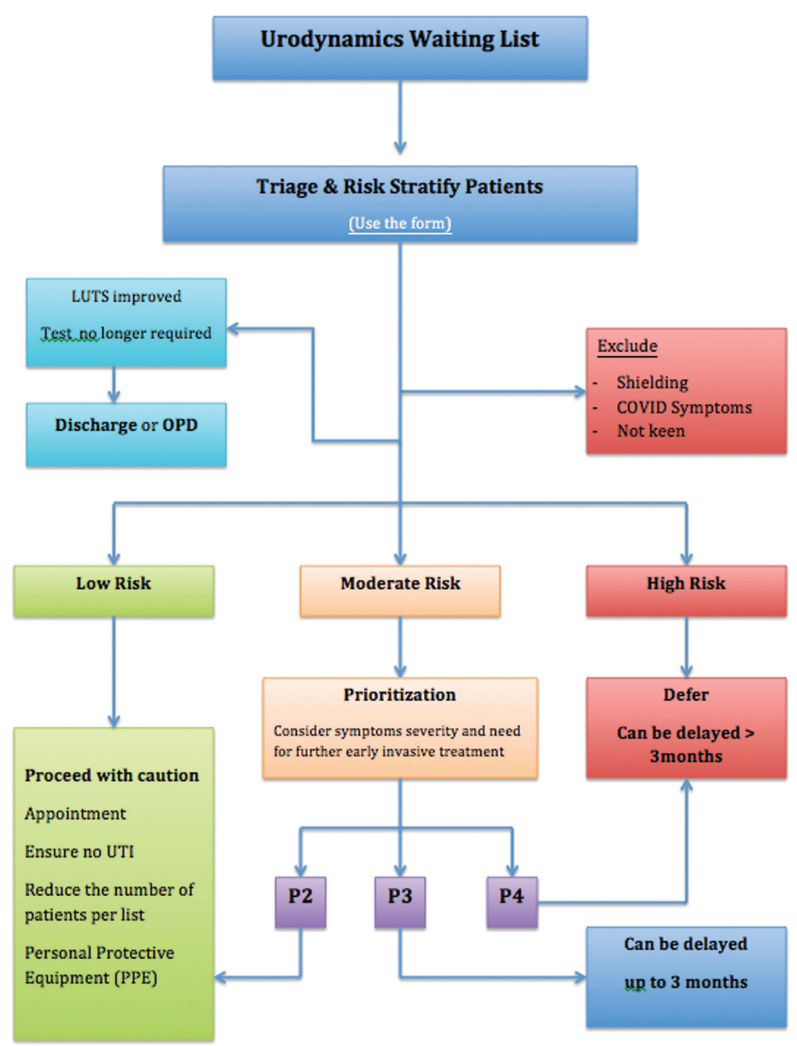


TABLE 1 Urodynamic Studies Triage Form

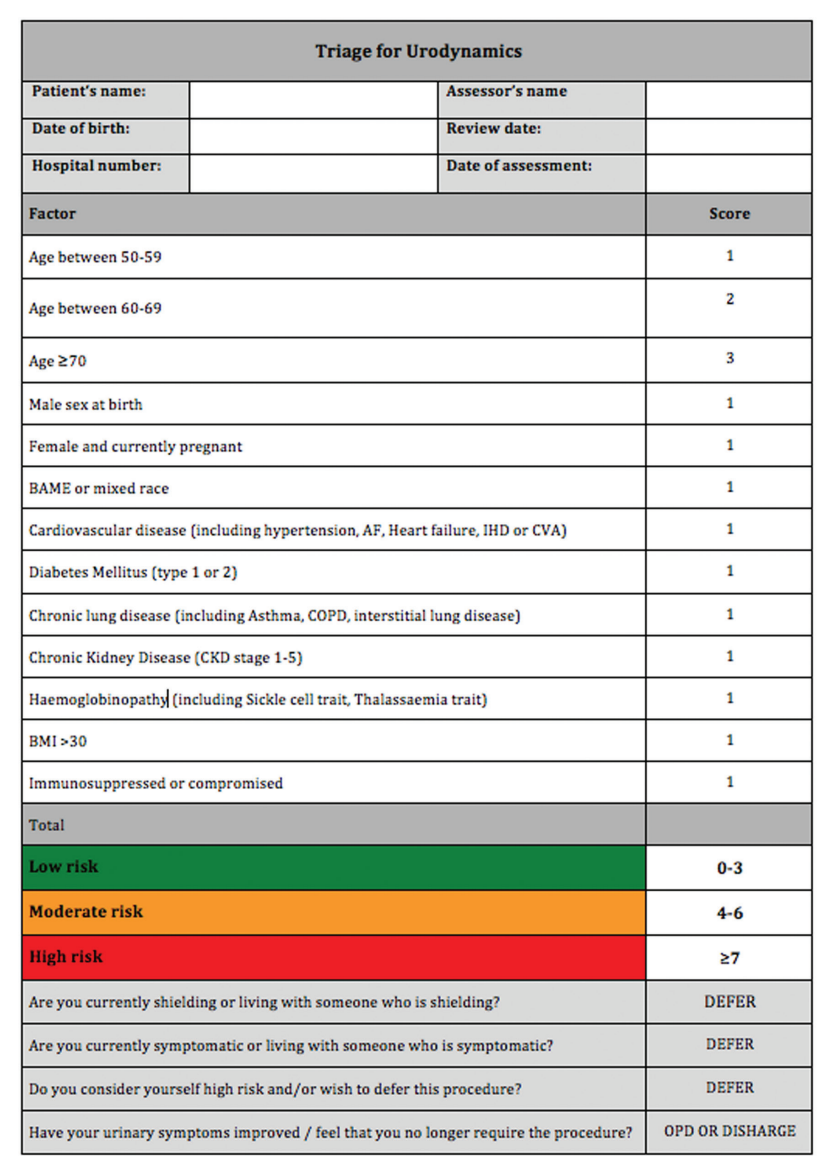

The authors hereby give consent to other colleagues to use this form and/or modify it to fit their local hospital needs and trust policy should they wish to adapt this approach in triaging patients before urodynamics.

\section{CONCLUSION}

This multicentre, international cohort study received 104 responses during two weeks following the distribution of the 14-point survey. It highlighted concerns from clinicians performing UDS, specifically relating to coughing and adequate PPE in light of the COVID-19 pandemic. It was evident that a lack of advice and guidance relating to safe practice when performing UDS caused uncertainty amongst clinicians. The authorship feels that careful patient selection for UDS and appropriate triage are necessary prerequisites for reinstating this important service. Furthermore, the authors have highlighted the potential need to explore and establish a reliable alternative method of urodynamic testing by substituting the cough-test with the Valsalva maneuver. However more collaborative research and high-level evidence will be needed to validate its accuracy in the future.

\section{REFERENCES}

1. Wein A, Kavoussi LR, Partin AW, et al. Campbell-Walsh Urology. Eleventh ed. Elsevier. c73. P1718-1733; 2016.

2. World Health Organisation. Novel Coronavirus (2019$\mathrm{nCoV}$ ). Situation Report - 1.21st Jan 2020. Available at: https://www.who.int/docs/default-source/coronaviruse/situation-reports/20200121-sitrep-1-2019-ncov. pdf?sfvrsn=20a99c10_4

3. World Health Organisation. Novel Coronavirus (2019-nCoV): Situation Report - 11. 31st Jan 2020. Available at: https://www.who.int/docs/default-source/ coronaviruse/situation-reports/20200131-sitrep-11-ncov. pdf?sfvrsn=de7c0f7_4

4. Public Health England. Guidance: Transmission Characteristics and Principles of Infection Prevention and Control. 2020. Last updated 18/06/2020. Available at: https://www.gov.uk/government/publications/wuhannovel-coronavirus-infection-prevention-and-control/ transmission-characteristics-and-principles-of-infectionprevention-and-control

5. World Health Organisation. WHO Coronavirus Disease (COVID-19) Dashboard. 2020. Last updated 03/07/2020. Available at: https://covid19.who.int/

6. Ribal MJ, Cornford P, Briganti A, et al. EAU Guidelines Office Rapid Reaction Group: An Organisation-Wide Collaborative Effort to Adapt the EAU Guidelines Recommendations to the COVID-19 era. 2020. https://uroweb.org/wp-content/uploads/ EAU-Guidelines-Office-Rapid-Reaction-Group-Anorganisation-wide-collaborative-effort-to-adapt-the-EAUguidelines-recommendations-to-the-COVID-19-era.pdf.

7. Lindsley WG, King WP, Thewlis RE, et al. Dispersion and exposure to a cough-generated aerosol in a simulated medical examination room. J Occup Environ Hyg 2013;10(2).

8. Young HS, Sun-Ouck K, Ho SO, et al. Leak point pressure at different bladder volumes in stress urinary incontinence in women: Comparison between Valsalva and cough-induced leak point pressures. Can Urol Assoc J 2016;10(1-2):E23-7. 
9. Iacobucci G. Covid-19: all non-urgent elective surgery is suspended for at least three months in England. BMJ 2020;368:m1106. Published 2020 Mar 18. doi:10.1136/ bmj.m1106

10. Hashim, H.; Thomas, L; Gammie, A.; et al. Good urodynamic practice adaptations during the COVID 19 pandemic. Neurourol Urodynamics 2020;1-5.

11. Rosier PM, Schaefer W, Lose G, et al. International Continence Society Good Urodynamic Practices and Terms 2016: Urodynamics, uroflowmetry, cystometry, and pressure flow study. 2016. Neurourol Urodynamics.

12. Sullivan J, Lewis P, Howell S, et al. Quality control in urodynamics: a review of urodynamic traces from one centre. BJU International 2003;91:201-207.

13. Lu T, Liao L. Typical value ranges and typical signal patterns in the initial cough in patients with neurogenic bladder: quality control in urodynamic studies. Int Neurourol J 2016;20(3):214-23.
14. The United States Government's Centre for Disease Control. Available at: https://www.cdc.gov/coronavirus/2019ncov/index.html

15. Department of Health and Social Care, United Kingdom Government. Update from the UK Chief Medical Officers on the UK alert level. Coronavirus (COVID-19): Guidance and Support. 2020. Available at: https://www.gov.uk/government/news/ update-from-the-uk-chief-medical-officers-on-the-uk-alert-level.

16. Public Health England. Guidance: COVID-19 personal protective equipment (PPE). Last updated 18/06/2020. Available at: https://www.gov.uk/government/publications/ wuhan-novel-coronavirus-infection-prevention-and-control/ covid-19-personal-protective-equipment-ppe.

17. Royal College of Surgeons of England: Tool 2 on Safety Recommendation and Risk Assessment. Published 14/05/2020. Available at https://www.rcseng.ac.uk/ coronavirus/recovery-of-surgical-services/tool-2/ 